\section{Zervix-Exzision: persistierende HPV-Infektion erhöht Rezidivrisiko}

Präkanzerosen und niedriggradige Karzinome der Zervix sind gut zu behandeln. In einer Kohortenstudie wurde der langfristige Verlauf und der Einfluss von Infektionen mit verschiedenen Subtypen des HPV (Human papillomavirus) in einer großen bevölkerungsbasierten Kohorte in Puerto Rico untersucht.

— ingeschlossen in die Untersuchung

— in den Jahren 2000 und 2001 waren Frauen aus Puerto Rico, die sich im Rahmen der Guanacaste Natural History Study einer Zervix-Exzisionsbehandlung unterzogen hatten. Die ursprüngliche Kohorte umfasste 10.049 Frauen. In der Nachuntersuchung wurden 681 Frauen mit verdächtigen Zervixbefunden zur Koloskopie überwiesen, 486 von ihnen wurden daraufhin als behandlungsbedürftig eingestuft und 347 Frauen in die Analyse eingeschlossen. HPV-Infektionen wurden als persistent eingestuft, wenn sie so- wohl vor als auch nach der Exzisionsbehandlung nachgewiesen wurden. Als Neuinfektionen wurden HPV-Nachweise gewertet, die lediglich nach der Behandlung detektiert wurden.

Die mediane Zeit zwischen der Zervix-Exzisionsbehandlung und den aktuellen Nachuntersuchungen betrug 6,7 Jahre. Bei den aktuellen Nachuntersuchungen hatten acht Frauen (2,4\%) eine persistierende HPV16-Infektion, zwei Frauen (0,6\%) eine persistierende HPV18-Infektion und acht $(2,4 \%)$ der 347 Frauen eine Infektion mit einem anderen karzinogenen HPV-Stamm. Neu- infektionen mit HPV16 wurden bei zwei Frauen (0,8 \%), mit HPV18 bei drei Frauen $(1,0 \%)$ und Infektionen mit anderen karzinogenen Stämmen bei 13 Frauen $(4,0 \%)$ detektiert. Bei den Nachbehandlungsterminen ergab sich bei sechs Frauen die Diagnose CIN2+. Alle Frauen hatten persistierende Infektionen (drei HPV16, eine HPV18 und zwei andere karzinogene HPV-Stämme). Bei keiner Frau mit einer Neuinfektion wurde ein Rückfall festgestellt.

Fazit: Frauen mit therapierter ZervixPräkanzerose bzw. niedriggradigem Zervixkarzinom haben binnen sechs Jahren nur selten eine persistierende HPV-Infektion. Wenn er aber vorliegt, erhöht ein persistierender Virusbefall das Risiko für CIN2+. Barbara Kerutzkamp

Kreimer AR et al. Long-term risk of recurrent cervical human papillomavirus infection and precancer and cancer following excisional treatment. Int J Cancer. 2012;131(1):211-8.

\title{
Impfung gegen HPV nach Virusexposition?
}

Die Impfung mit dem humanen, rekombinanten und adjuvantierten PapillomImpfstoff Cervarix ${ }^{\circledR}$ gegen die Virustypen 16/18 erwies sich in der PATRICIAStudie bei jungen Frauen ohne vorherigen Viruskontakt als hoch effektiv gegen HPV-16/18-Infektionen und präkanzeröse Läsionen im Gebärmutterhals. In einer aktuellen Studie wurde untersucht, ob auch Frauen nach einer HPV-16- und/oder -18-Exposition von dieser Impfung profitieren.

$\mathrm{n}$ der doppelblinden, multizentrischen, randomisierten und kontrollierten Phase-III-Studie PATRICIA - mit 18.644 Teilnehmerinnen im Alter zwischen 15 und 25 Jahren aus 14 Ländern eine der größten ihrer Art - wurde die Wirksamkeit der HPV-Vakzine gegen Zervixdysplasien und -karzinome in Zusammenhang mit einer HPV-16/18-Infektion untersucht. Die Kontrollgruppe erhielt einen Hepatitis-A-Impfstoff. Frauen ohne Virus-DNA im Zervixabstrich, die vor der Erstimpfung seronegativ für VirusAntikörper waren, waren in der Primäranalyse nach rund vier Jahren Follow-up zu 92,9\% gegen zervikale intraepitheliale Neoplasien vom Grad 2 oder höher (CIN2+) geschützt. Die Autoren erweiterten die PATRICIA-Studie um die Analyse des möglichen Einflusses einer früheren Exposition gegen die onkoge- nen HPV-Typen 16/18 auf die Stärke des Impfschutzes (VE = Vaccine Efficacy). Die Teilnehmerinnen wurden entsprechend ihrer Histopathologie und ihres serologischen Status bei Studienbeginn (Baseline) in drei Gruppen stratifiziert und die VE ermittelt.

Gruppe 1: DNA-negativ für HPV-16/18 unabhängig vom serologischen Status Gruppe 2: DNA-negativ für HPV-16/18, aber seropositiv für HPV-16 und/oder -18 Gruppe 3: DNA-negativ für einen HPVTyp, DNA-positiv für den anderen

Ergebnisse zu den ausgewählten Endpunkten,,sechsmonatige persistierende Infektion (PI)“ und CIN2+ in den drei Gruppen:

1: VE von $88,7 \%$ bzw. 92,4\%

2: VE von $72,3 \%$ bzw. $68,8 \%$

3: $\mathrm{VE}$ von $90 \%$ gegen den Endpunkt "CIN2+"
Die weiterführende Analyse der PATRICIA-Daten belegt die hohe Wirksamkeit der Anti-HPV-16/18-Vakzine gegen virologische und histopathologische Endpunkte bei Viren-DNA-negativen Frauen unabhängig vom serologischen Status bei Studienbeginn, so die Autoren. Der Impfschutz war bei serologischen Hinweisen auf eine frühere Infektion substanziell, wenngleich abgeschwächt. Außerdem sind Frauen mit bestätigter Infektion mit einem der genannten $\mathrm{Vi}$ rustypen nach der Impfung effektiv vor der zweiten, vom Impfstoff abgedeckten Virusvariante geschützt.

Fazit: Auch Frauen mit serologisch nachgewiesener früherer HPV-16/18-Infektion profitieren von einer Immunisierung mit dem HPV-16/18-Impfstoff, wenn auch in geringerem Ausmaß als HPV-16/18-Naive. Die Autoren raten, auch nach der Impfung weiter an Vorsorgeuntersuchungen teilzunehmen.

Wolfgang Zimmermann

Szarewski A et al. Efficacy of the human papillomavirus (HPV)-16/18 AS04-adjuvanted vaccine in women aged 15-25 years with or without serological evidence of previous exposure to HPV16/18. Int J Cancer. 2012;131(1):106-16. 